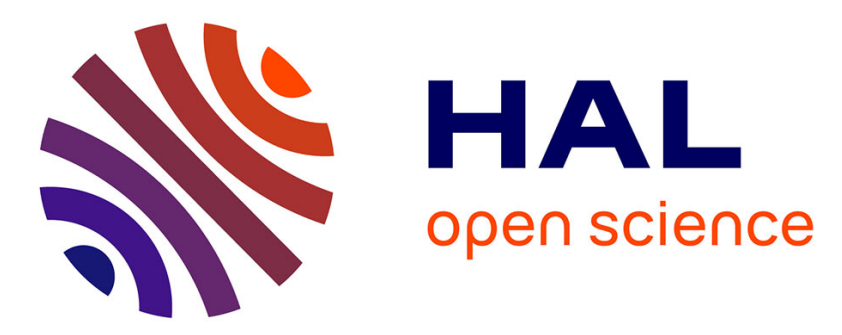

\title{
Analysis of clarinet reed oscillations with digital Fresnel holography
}

P. Picart, J. Leval, F. Piquet, J.-P. Boileau, Jean-Pierre Dalmont

\section{To cite this version:}

P. Picart, J. Leval, F. Piquet, J.-P. Boileau, Jean-Pierre Dalmont. Analysis of clarinet reed oscillations with digital Fresnel holography. European Physical Journal: Applied Physics, 2009, 47 (1), pp.1-8. 10.1051/epjap/2008201 . hal-00480142

\section{HAL Id: hal-00480142 \\ https://hal.science/hal-00480142}

Submitted on 3 May 2010

HAL is a multi-disciplinary open access archive for the deposit and dissemination of scientific research documents, whether they are published or not. The documents may come from teaching and research institutions in France or abroad, or from public or private research centers.
L'archive ouverte pluridisciplinaire HAL, est destinée au dépôt et à la diffusion de documents scientifiques de niveau recherche, publiés ou non, émanant des établissements d'enseignement et de recherche français ou étrangers, des laboratoires publics ou privés. 


\title{
Analysis of Clarinet Reed Oscillations With Digital Fresnel Holography
}

\author{
*Pascal Picart ${ }^{1,2}$ \\ phone : 0033.2.43.83.39.58., fax : 0033.2.43.83.37.94., e-mail : pascal.picart@ univ-lemans.fr \\ Julien Leval ${ }^{1}$ \\ phone : 0033.2.43.83.39.55., fax : 0033.2.43.83.37.94., e-mail : julien.leval@univ-lemans.fr \\ Francis Piquet ${ }^{2}$ \\ phone : 0033.2.43.83.34.59., fax : 0033.2.43.83.31.49., e-mail : francis.piquet@univ-lemans.fr \\ Jean Pierre Boileau ${ }^{2}$ \\ phone : 0033.2.43.83.39.52., fax : 0033.2.43.83.37.94., e-mail : jean-pierre.boileau@ univ-lemans.fr \\ Jean Pierre Dalmont ${ }^{1}$ \\ phone : 0033.2.43.83.32.77., fax : 0033.2.43.83.35.20., e-mail : jean-pierre.dalmont@univ-lemans.fr \\ * corresponding author \\ e-mail : pascal.picart@univ-lemans.fr \\ 1. LAUM, CNRS, Université du Maine, Av. O. Messiaen, 72085 LE MANS, France. \\ 2. ENSIM-École Nationale Supérieure d'Ingénieurs du Mans, rue Aristote, 72085 LE MANS, France.
}

Abstract - This paper describes optical instrumentation devoted to vibration analysis. Two strategies based on digital Fresnel holography are presented. The first one, called time-averaging consists in the numerical reconstruction of the hologram after recording with an exposure time much greater than the vibration period. Thus, the holograms have their amplitude modulated by a Bessel function. The last strategy, using a pulsed regime, allows the reconstruction of the full movement of the vibration, even if it exhibits very high amplitude. Experimental results presented in this paper concern the study of the vibrations of a clarinet reed under free and forced oscillation regimes.

Keywords - digital holography, holographic interferometry, interferometry, instrumentation measurement and metrology, optical inspection, acoustics, musical acoustics

PACS numbers: 07.60.Ly; 42.40.-I; 42.40.Kw 


\section{INTRODUCTION}

Digital holography became properly available since its confirmation was established in 1994 [1]. In few years, a lot of wonderful applications have been demonstrated such as digital holographic microscopic imaging [2], securing information [3], surface shape measurements [4], remote metrology [5] and vibrations analysis [6-9]. In vibration analysis, time averaging [7] was demonstrated and it appears that it is a very useful tool to investigate the properties of objects under sinusoidal excitation. However, the vibration is observed in the sense of its amplitude since its phase is lost during the process. So, pseudo-pulsed digital holography was developed for reconstructing both amplitude and phase of the vibration [8]. An extension of this principle into simultaneous 2D full field vibration analysis by combining digital holography and spatial multiplexing of holograms was also proposed [9]. However such a strategy is only valid for sinusoidal vibrations. Pulsed lasers are also used for reconstructing vibrations [6]. This allows freezing the object at the time of the recording. Generally, experimental results are limited to double pulse experiments, that means vibration is not fully reconstructed in the sense of its amplitude and phase. Considering that, this paper aims to present a thorough investigation of vibrations by using digital Fresnel holography. The investigation is applied to a clarinet reed. The reed is excited following two modes: a pure monochromatic sinusoidal regime and a pure autooscillation regime. Two interferometers were constructed allowing studies with the time averaging principle and the full reconstruction of the vibration using a pulsed light beam. The paper is organized as follows: Sections 2 describes the basic principle of digital Fresnel holography, in Section 3 different strategies for vibration analysis will be discussed, Section 4 presents the experimental setups developed for the study of forced and free oscillations of a clarinet reed. Section 5 draws the conclusions of the study. 


\section{THEORETICAL BASICS}

Consider a reference set of coordinate $\{x, y, z\}$ attached to the surface of an object of interest, $z$ being perpendicular to the surface and considered as the propagation direction of the diffracted light beam (see figure 1 for example). The object surface illuminated by a coherent beam of wavelength $\lambda$ produces an object wave front noted $A(x, y)=A_{0}(x, y) \exp \left[j \psi_{0}(x, y)\right]$. Phase $\psi_{0}$ is random because of the roughness of the surface and it will be considered that it is uniform over $[-\pi,+\pi]$. In what follows, it is considered that the object wave propagates through a distance $d_{0}$ at which it interferes with a plane and smooth reference wave having spatial frequencies noted $\left\{u_{R}, v_{R}\right\}$. At distance $d_{0}$, the reference set of coordinate is chosen to be $\left\{x^{\prime}, y^{\prime}, z\right\}$. The diffracted field produced by the temporally varying object is given in the Fresnel approximations by $[10,11]$

$$
\begin{aligned}
O\left(x^{\prime}, y^{\prime}, d_{0}, t\right)= & \frac{i \exp \left(i 2 \pi d_{0} / \lambda\right)}{\lambda d_{0}} \exp \left[\frac{i \pi}{\lambda d_{0}}\left(x^{\prime 2}+y^{\prime 2}\right)\right] \\
& \times \int_{-\infty}^{+\infty} \int_{-\infty}^{+\infty} A(x, y, t) \exp \left[\frac{i \pi}{\lambda d_{0}}\left(x^{2}+y^{2}\right)\right] \exp \left[-\frac{2 i \pi}{\lambda d_{0}}\left(x x^{\prime}+y y^{\prime}\right)\right] d x d y
\end{aligned}
$$

The reference wave can be simply described using a spatially biased phase:

$$
R\left(x^{\prime}, y^{\prime}\right)=a_{R} \exp \left[2 j \pi\left(u_{R} x^{\prime}+v_{R} y^{\prime}\right)\right]
$$

In the interference plane, the temporally varying hologram $H$ is written:

$$
\begin{aligned}
H\left(x^{\prime}, y^{\prime}, d_{0}, t\right) & =\left|O\left(x^{\prime}, y^{\prime}, d_{0}, t\right)\right|^{2}+\left|R\left(x^{\prime}, y^{\prime}\right)\right|^{2} \\
& +R^{*}\left(x^{\prime}, y^{\prime}\right) O\left(x^{\prime}, y^{\prime}, d_{0}, t\right)+R\left(x^{\prime}, y^{\prime}\right) O^{*}\left(x^{\prime}, y^{\prime}, d_{0}, t\right)
\end{aligned}
$$

In digital Fresnel holography, the interferogram is recorded with a matrix of pixels thus discretizing the spatial coordinate in the recording plane. Generally, the detector includes $M \times N$ pixels of pitches $p_{x}$ and $p_{y}$, each of them sized $\Delta_{x} \times \Delta_{y}$. Furthermore, in the case of a time-varying hologram, the hologram effectively recorded by the sensor during the 
exposure time $T$ is given by:

$H\left(x^{\prime}, y^{\prime}, d_{0}, T\right)=\int_{t_{1}}^{t_{1}+T} H\left(x^{\prime}, y^{\prime}, d_{0}, t\right) d t$.

The object wave can be digitally reconstructed by using a discrete version of the Fresnel integral (equation (1)). The numerically reconstructed object will be in focus for a reconstruction distance equal to $d_{R}=-d_{0}$ in the reconstruction algorithm. Thus, the diffracted field in the +1 order, noted $A_{R}^{+1}$, at distance $d_{R}=-d_{0}$ from the recording plane can be computed with $(K, L) \geq(N, M)$ data points by evaluating the following equation [9] :

$$
\begin{aligned}
A_{R}^{+1}\left(X, Y,-d_{0}\right)= & -\frac{j \exp \left(-2 j \pi d_{0} / \lambda\right)}{\lambda d_{0}} \exp \left[-\frac{j \pi}{\lambda d_{0}}\left(X^{2}+Y^{2}\right)\right] \\
& \times \sum_{k=0}^{k=K-1 l=L-1} \sum_{l=0} H\left(k p_{x}, l p_{y}, d_{0}, T\right) \exp \left[-\frac{j \pi}{\lambda d_{0}}\left(k^{2} p_{x}^{2}+l^{2} p_{y}^{2}\right)\right] . \\
& \times \exp \left[+\frac{2 j \pi}{\lambda d_{0}}\left(k p_{x} X+l p_{y} Y\right)\right]
\end{aligned}
$$

Note that in the case where $(K, L) \geq(N, M)$ the digital hologram will be padded with $(K-N, L-M)$ zeros [12]. In reference [7] was demonstrated that the diffracted field in +1 order is given by (* means convolution):

$$
\begin{aligned}
A_{R}^{+1}\left(X, Y,-d_{0}\right) & =\lambda^{2} d_{0}^{2} \exp \left[-j \pi \lambda d_{0}\left(u_{R}^{2}+v_{R}^{2}\right)\right] R^{*}(X, Y) \\
& \times A_{T}(X, Y) * \tilde{W}_{N M}\left(X, Y,-d_{0}\right) * \delta\left(X-\lambda u_{R} d_{0}, Y-\lambda v_{R} d_{0}\right)
\end{aligned}
$$

where $\tilde{W}_{N M}\left(X, Y,-d_{0}\right)$ is the filtering function of bi-dimensional discrete Fourier transform, given by [7]

$$
\begin{aligned}
\tilde{W}_{N M}\left(x, y,-d_{0}\right) & =\exp \left[-j \pi(N-1) \frac{x p_{x}}{\lambda d_{0}}-j \pi(M-1) \frac{y p_{y}}{\lambda d_{0}}\right] \\
& \times \frac{\sin \left(\pi N x p_{x} / \lambda d_{0}\right)}{\sin \left(\pi x p_{x} / \lambda d_{0}\right)} \frac{\sin \left(\pi M y p_{y} / \lambda d_{0}\right)}{\sin \left(\pi y p_{y} / \lambda d_{0}\right)}
\end{aligned}
$$


Note that $A_{T}(X, Y)$ is the real object temporally integrated by the detector. Two regimes may be considered for the recording: time averaging regime and pulsed regime. If the hologram is recorded in time averaging regime when the object is excited with a pure sinusoidal excitation (frequency $f_{0}, T \gg 1 / f_{0}$ ), the reconstructed object appears to be the real object whose amplitude is modulated by the Bessel function $J_{0}$ according to [7]:

$$
\left|A_{T}(X, Y)\right|=\left|J_{0}\left(\Delta \varphi_{m}(X, Y)\right)\right|\left|A_{0}(X, Y)\right|,
$$

where $\Delta \varphi_{m}$ is the maximum amplitude of the sinusoidal optical phase variation due to the oscillation of the object. The Bessel modulation indicates the modal structure of the object under the sinusoidal excitation.

In the opposite case, ie the hologram is recorded with a pulse light shorter than the oscillation period of the object, then one gets $H\left(x^{\prime}, y^{\prime}, d_{0}, T\right) \approx H\left(x^{\prime}, y^{\prime}, d_{0}, t_{j}\right)$ with $t_{j}$ being the time at which is started the recording, then we have [8]

$\left.A_{T}(X, Y)=A_{0}(X, Y) \exp \mid i \psi_{0}\left(X, Y, t_{j}\right)\right\rfloor$

In this last case, the variations of the optical phase $\psi_{0}$ between several recordings allow to reconstruct the full movement of the excited object, knowing the sensitivity vector of the interferometric setup. The next section discusses the investigation of vibrations using digital Fresnel holography.

\section{INVESTIGATION OF VIBRATIONS}

For the analysis of vibrations, one must distinguish between free and forced oscillations. In forced oscillation regime, the amplitude of vibrations can be controlled by the excitation source whereas in the free oscillation regime the object imposes its own rule on the observer. So, the digital holographic setup must be configured according to the amplitude of the oscillation. In the time-averaging regime one particularity of the setup is 
that the vibration is under a monochromatic excitation. This means that the maximum amplitude of oscillation to be measured can be controlled and chosen so that the number of fringes due to the Bessel modulation in reconstructed holograms is low (equation (8)). This way for analysing vibrations is quite simple and doesn't need a complicated setup although the experimental results are partial because of the disappearance of the vibration phase in the reconstructed object [7]. Nevertheless, as a general rule, acoustics engineers are often confronted with free oscillations which are multi-chromatic and have high amplitude regarding the optical wavelength. This kind of vibration is generally not controlled since it is often initiated with nonlinear phenomena leading to an autooscillation phenomenon. The study of such oscillation needs a more elaborated strategy than that used for forced oscillations. Indeed, the oscillation must be finely temporally sampled with a temporal resolution allowing the fringe resolution in the phase data, especially is the amplitude is high. The full movement of the object can then be reconstructed by adding all the partial results obtained with the temporal sampling. Note that the greater the oscillation amplitude is, greater must be the number of recorded holograms. An example of such vibrations is found in a single-reed woodwind musical instrument. The clarinet is typically such an instrument. Its reed is a small slice of natural cane or synthetic material put into vibration when the musician is blowing air into the instrument. The clarinet is usually considered as the association of a linear resonator, the pipe, and a nonlinear exciter, the reed, subject to the air flow from the mouth. The role of the lowest reed resonance frequency in sound production by single-reed instruments was established in the past [13]. The understanding of the physical mechanisms that lead to the production of sound in the clarinet needs an in-depth investigation of the reed oscillation under monochromatic excitation and under the free oscillation regime. So the experimental investigation of the clarinet reed behaviour was performed with the 
development of two digital holographic interferometers allowing the visualisation of the reed movement with time averaging holograms and with pulsed holograms.

\section{EXPERIMENTAL SETUPS AND RESULTS}

The holographic interferometers are based on Mach-Zehnder architecture with off-axis recordings [7-9]. The laser is chosen according to the oscillation regime: a continuous HeNe laser $(\lambda=632.8 \mathrm{~nm})$ for time-averaging and a frequency doubled NdYAG pulsed laser $(\lambda=532 \mathrm{~nm})$ for the free oscillation regime. The reference wave is issued from a spatial filter in order to be smooth, plane and with uniform amplitude. The sensor is a digital camera with $\{M \times N\}=\{1024 \times 1360\}$ pixels of pitches $p_{x}=p_{y}=4.65 \mu \mathrm{m}$ (PCO Pixel Fly). The numerical reconstruction of the holograms is performed according to equation (5) with a two-dimensional discrete Fourier transform. The clarinet reed is placed at a distance which depends on the size of the inspected zone. For a rectangular zone of size $(a \times b)$, larger size is vertical, $a>b$, the distance at which must be placed the object is given by [14]:

$d_{0}=\frac{4 p_{y} a}{\lambda}$

and the couple of optimal spatial frequencies for the reference wave are given by

$$
\left(u_{R}, v_{R}\right)=\left( \pm \frac{3}{8 p_{y}}, 0\right)
$$

The optimal values of these parameters allow an optimal recording in which the three orders do not overlap and the object is fully included in the diffracted field.

\subsection{CASE OF TIME AVERAGING}

For the forced oscillation regime the inspected zone is about $(a \times b)=(35 \mathrm{~mm} \times 9 \mathrm{~mm})$. Thus the recording distance is set to be $d_{0}=1030 \mathrm{~mm}$. In forced oscillation regime, the clarinet 
reed is submitted to an acoustic wave emitted by a loudspeaker placed behind the reed. In the set-up, the clarinet reed is fixed with a ligature as it is the case in usual conditions. The amplitude and the frequency of the loudspeaker can be controlled so that the number of fringes in the Bessel modulation remains relatively low, ie the fringes are resolvable. The laser power is adjusted such that the exposure time of the detector is $T=1 \mathrm{~s}$, ensuring the condition $T>>1 / f_{0}$ for $f_{0}>100 \mathrm{~Hz}$. Figure 1 shows the experimental setup for excitation and recording in the time averaging regime. Figure 2 and 3 show the vibration modes of the clarinet reed in the frequency range $1 \mathrm{kHz}-10 \mathrm{kHz}$. It can be observed that the first and the second pure bending mode at $1880 \mathrm{~Hz}$ and $4500 \mathrm{~Hz}$, the first torsion mode at $3260 \mathrm{~Hz}$ and other modes which are characteristic of the vibration behaviour of this reed. These modal structures are indicators of mechanical properties of the reed; it can be expected that future work will allow to qualify the aptitude of any reed for the musical playing.

\subsection{CASE OF FREE OSCILLATIONS}

As pointed out previously, the case of free oscillations for the clarinet reed corresponds to playing conditions, i.e. when the clarinet reed is in the autooscillation regime. In order to produce suitable oscillation conditions, an artificial mouth modeling that of a musician playing clarinet was built in the laboratory [15]. This artificial mouth is constituted mainly with an air-proof caisson and an artificial lip modeling the tight-lipped going of the musician. The schematic diagram of the artificial mouth is depicted in figure 4 . In the setup, the clarinet reed is fixed with a ligature as it is the case in usual conditions. The artificial lip is made with a rubber membrane filled with water so that it has quite the same consistency as the real musician's lip. The mouth includes an optical window which allows illumination of the reed. To initiate free oscillation of the reed, flow is blown in the mouth. The overpressure in the mouth is of the order of $60 \mathrm{hPa}$. It induces a flexure of the reed and introduces an air flow into the instrument. Hereafter a threshold pressure, the 
equilibrium position of the reed becomes unstable and the reed oscillates at a frequency inversely proportional to the effective length of the clarinet. The sound generated by this oscillation is periodic but multi-chromatic and in the setup the fundamental frequency was about $162 \mathrm{~Hz}$. The particularity of the observed autooscillation regime, classically called beating reed regime, is that the amplitude is imposed by the characteristics of the mouthpiece. This amplitude can vary from a few tenths of millimetre to one millimetre. A pressure sensor made with piezo-resistive gages (microphone) is placed in the mouthpiece of the clarinet, as indicated in figure 4 (top view). This sensor measures the pressure fluctuations induced by the mechanical vibration of the reed. The pressure signal delivered by the mouth is of primary use for the optical setup described in figure 5 . The laser beam is issued from the double frequency pulsed NdYAG laser with a pulse width of $20 \mathrm{~ns}$ and pulse energy about $7 \mathrm{~mJ}$. The inspected zone is a square region of about $(9 \mathrm{~mm} \times 9 \mathrm{~mm})$, thus, the mouth is placed in such a way that the reed is at about $d_{0}=315 \mathrm{~mm}$ from the detector area. In the set-up, illuminating angle $\theta$ is set to $22^{\circ}$ for experimental convenience. Figure 6 shows a photograph of the artificial mouth illuminated by the pulsed laser. Considering that the amplitude can reach several hundredths of microns, the study of the vibrating field must be performed according to a stroboscopic strategy. The laser firing is adjusted such that the movement is finely sampled giving resolved fringes in the field of view. Thus the pressure sensor included in the beak gives a reference signal which is used to synchronise the laser firing and the digital recording. The reference instant is the zero crossing of the pressure signal, then the laser firing is temporally shifted from $k \Delta \tau+k^{\prime} / f_{0}$. Integer $k^{\prime}$ indicates the number of oscillation period before the next laser firing and integer $k$ gives the temporal delay between two consecutive samplings of the oscillation. The recording mode takes into account of the maximum cadence of the sensor ( $7 \mathrm{~Hz}$ ). Results presented in the paper are obtained with $k^{\prime}=28$ and $\Delta \tau=2 \mu$ s and 3150 
digital holograms were recorded and computed for reconstructing the full movement of the reed during one oscillation period. Note that the time for the full data processing is about 24 hours. A graphical interface manages, via a microprocessor, all the parameters: pressure signal, charge of the flash pump of the laser, switch of the Pockels cell in the laser and trigger of the sensor. Figure 7 shows a period of the synchronization signal. It exhibits four important parts during one period of the free oscillation. The first part is called the opening reed since the reed is in a high level position regarding to the mouthpiece. Then, the second part corresponds to the closing phase during which the reed goes tight to the mouthpiece. The third part corresponds to the closing of the reed: the reed closes the reed channel. After that, the last part of the cycle corresponds to the opening phase during which the reed goes to its high level position. So, the movement of the reed is bi-stable. Figures 8 to 13 present experimental results of the reed movement for six time intervals indicated in figure 7 by points $\{A, B, C, D, E, F, G, H, I, J, K, L\}$. In some figures, some shapes (see for example figure 12) feature high irregularity while others don't (see for example figure 10). The reason is due to the amplitude shown in the figure. Especially, high amplitude reconstructed with a large number of holograms exhibit less noise than small amplitude reconstructed with a small number of holograms. Note that the signal of figure 7 is not exactly that of the laser sampling since the effective acquisition rate is $5.63 \mathrm{~Hz}$. Consequently the reconstructed movement is not the real displacement of the reed but it corresponds to a synthetic displacement constituted with sampling data points temporally spaced from $177.62 \mathrm{~ms}$. On figure 7 , points A to E correspond to open valve, this means the reed is in its higher position. Points $\mathrm{E}$ and $\mathrm{F}$ correspond to the closing phase, and then from $\mathrm{F}$ to $\mathrm{L}$ one observes the phase during which the reed goes to the beak to close the valve. Figures 8 to 13 show the deformation of the reed and the inspected zone included 138490 data points. The color map gives the relative 
displacement in micron units. Figures 11, 12 exhibit shocks of the reed. These shocks induce a strong deformation of the reed. To the best of our knowledge, these results constitute a first attempt to reconstruct a synthetic reed deformation on a pseudo-period of its autooscillation regime in an artificial mouth.

\section{CONCLUSION}

This paper presents methods for vibration analysis using digital Fresnel holography. Methods are based on time averaging for forced oscillations and pulsed recording devoted to free oscillations. The two methods are applied to a clarinet reed. In the forced oscillation regime, the reed is excited by an acoustic wave with controlled frequency and amplitude such that the reconstructed holograms exhibit resolvable Bessel fringes. These Bessel fringes are a signature of the mechanical behaviour of the reed. In the case of the autooscillation regime, the reed is placed in an artificial mouth. Deformation of the clarinet reed can be extracted with the recording of 3150 pulsed digital holograms virtually temporally spaced of $2 \mu \mathrm{s}$. The method allows high spatial resolution on the reconstructed image. Experimental results show the vibration behaviour of the clarinet reed under autooscillation regime, exhibiting high amplitude shocks during the closed phase. These works give opportunity to acoustics researchers to better understand sound production and musical quality of a clarinet.

\section{ACKNOWLEDGMENT}

The authors are very grateful to the reviewers for their careful reading and their useful remarks.

\section{REFERENCES}

1. U. Schnars, W. Jüptner, App. Opt. 33, 179 (1994)

2. E. Cuche, F. Bevilacqua, C. Depeursinge, Opt. Lett. 24, 291 (1999) 
3. B. Javidi, T. Nomura, Opt. Lett. 25, 28 (2000)

4. I. Yamaguchi, T. Ida, M. Yokota, K. Yamashita, App. Opt. 45, 7610 (2006)

5. T. Baumbach, W. Osten, C. von Kopylow, W. Juptner, App. Opt. 45, 925 (2006)

6. T. Saucedo, F.M. Santoyo, M. De la Torre Ibarra, G. Pedrini, W. Osten, App. Opt. 45, $4534(2006)$

7. P. Picart, J. Leval, D. Mounier, S. Gougeon, Opt. Lett. 28, 1900 (2003)

8. J. Leval, P. Picart, J.-P. Boileau, J.-C. Pascal, App. Opt. 44, 5763 (2005)

9. P. Picart, J. Leval, M. Grill, J.-P. Boileau, J.C. Pascal, J.-M. Breteau, B. Gautier, S. Gillet, Opt. Expr. 13, 8882 (2005)

10. Th. Kreis, M. Adams, W. Jüptner, Proc. SPIE 3098, 224 (1997)

11. J.W. Goodman, Introduction to Fourier Optics, $2^{\text {nd }}$ Edition (McGraw-Hill Editions, New York, 1996)

12. P. Ferraro, S. De Nicola, A. Finizio, G. Pierattini, G. Coppola, Appl. Phys. Lett. 85, 2709 (2004)

13. S. C. Thompson, J. Acoust. Soc. Am. 66, 1299 (1979)

14. P. Picart, J. Leval, JOSA A 25, 1744 (2008)

15. J.P. Dalmont, J. Gilbert, J. Kergomard, S. Ollivier, J. Acoust. Soc. Am. 118, 3294 (2005) 


\section{Captions}

Figure 1. Digital holographic interferometer for the time averaging regime

Figure 2. Time averaged holograms of the reed in the frequency range $1 \mathrm{kHz}-5 \mathrm{kHz}$

Figure 3. Time averaged holograms of the reed in the frequency range $5 \mathrm{kHz}-10 \mathrm{kHz}$

Figure 4. Artificial mouth

Figure 5. Digital holographic interferometer for the free oscillation regime

Figure 6. Photograph of the artificial mouth illuminated by the pulsed laser

Figure 7. One period of the pressure signal issued from the mouth

Figure 8. Reed deformation for $\Delta t=0.340 \mathrm{~ms}$ between A and B

Figure 9. Reed deformation for $\Delta t=1.416 \mathrm{~ms}$ between $\mathrm{C}$ and $\mathrm{D}$

Figure 10. Reed deformation for $\Delta t=0.654 \mathrm{~ms}$ between $\mathrm{E}$ and $\mathrm{F}$

Figure 11. Reed deformation for $\Delta t=0.922 \mathrm{~ms}$ between $\mathrm{G}$ and $\mathrm{H}$

Figure 12. Reed deformation for $\Delta t=0.944 \mathrm{~ms}$ between I and J

Figure 13. Reed deformation for $\Delta t=0.862 \mathrm{~ms}$ between $\mathrm{K}$ and $\mathrm{L}$ 


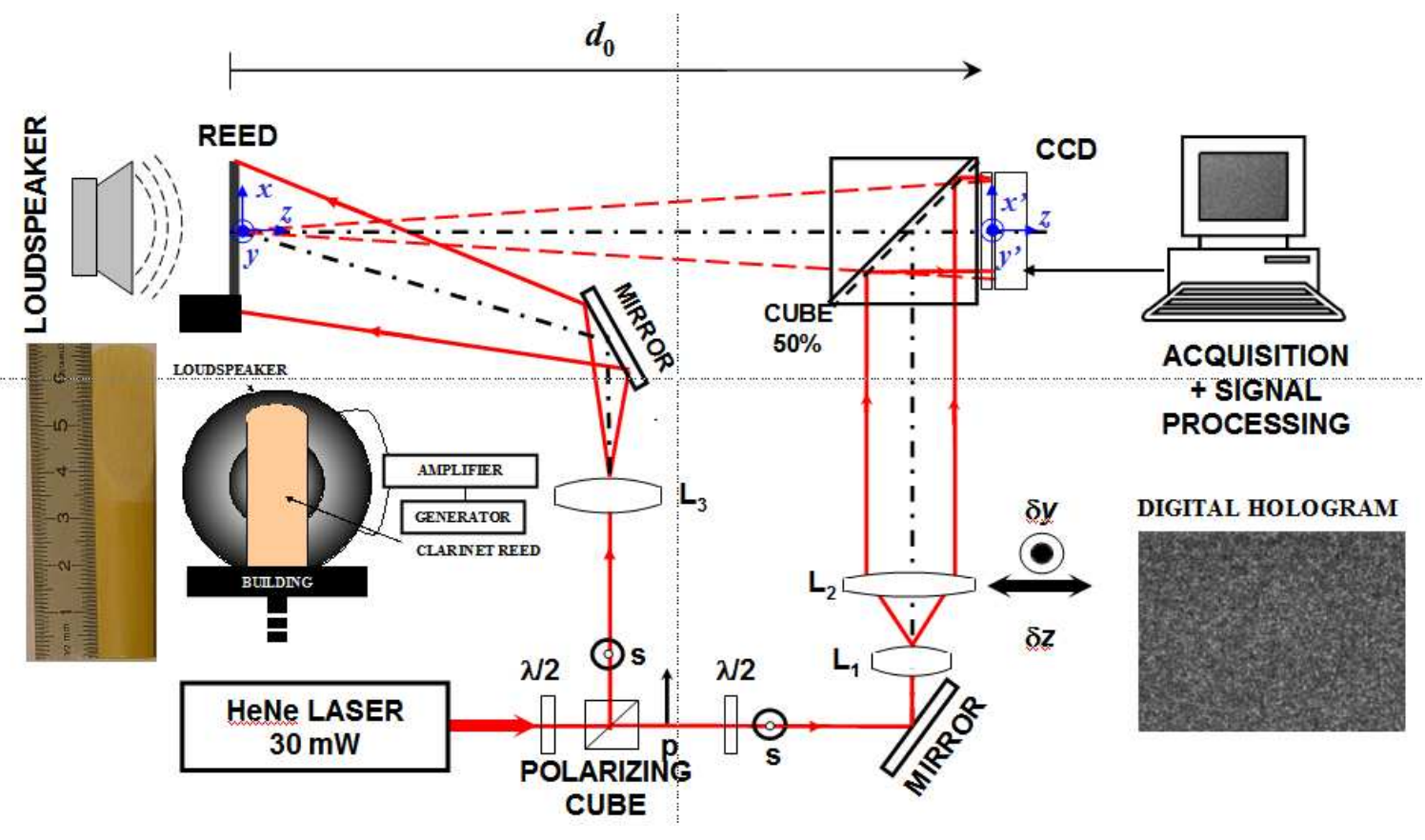

Figure 1 


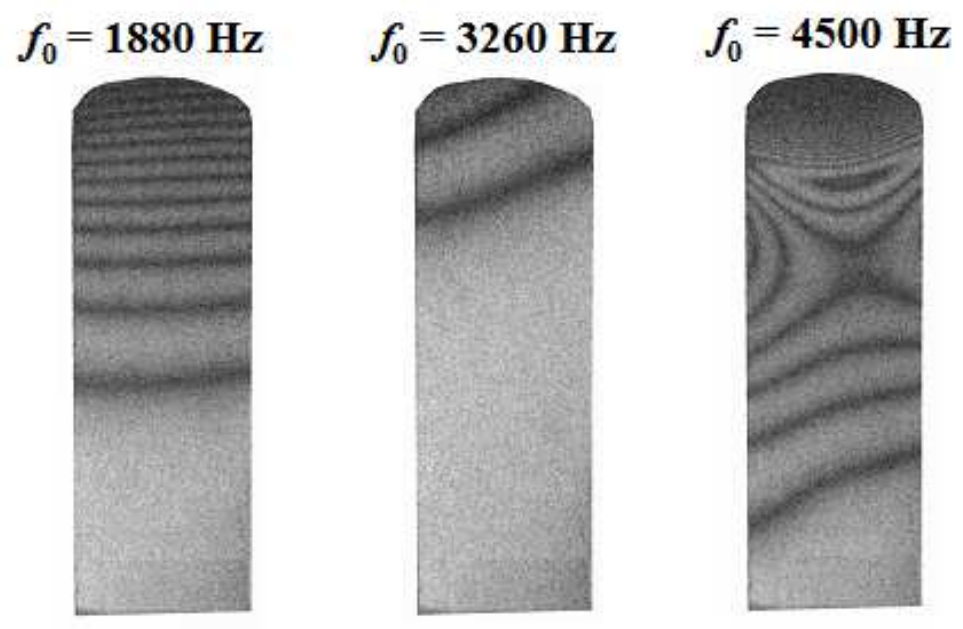

Figure 2 


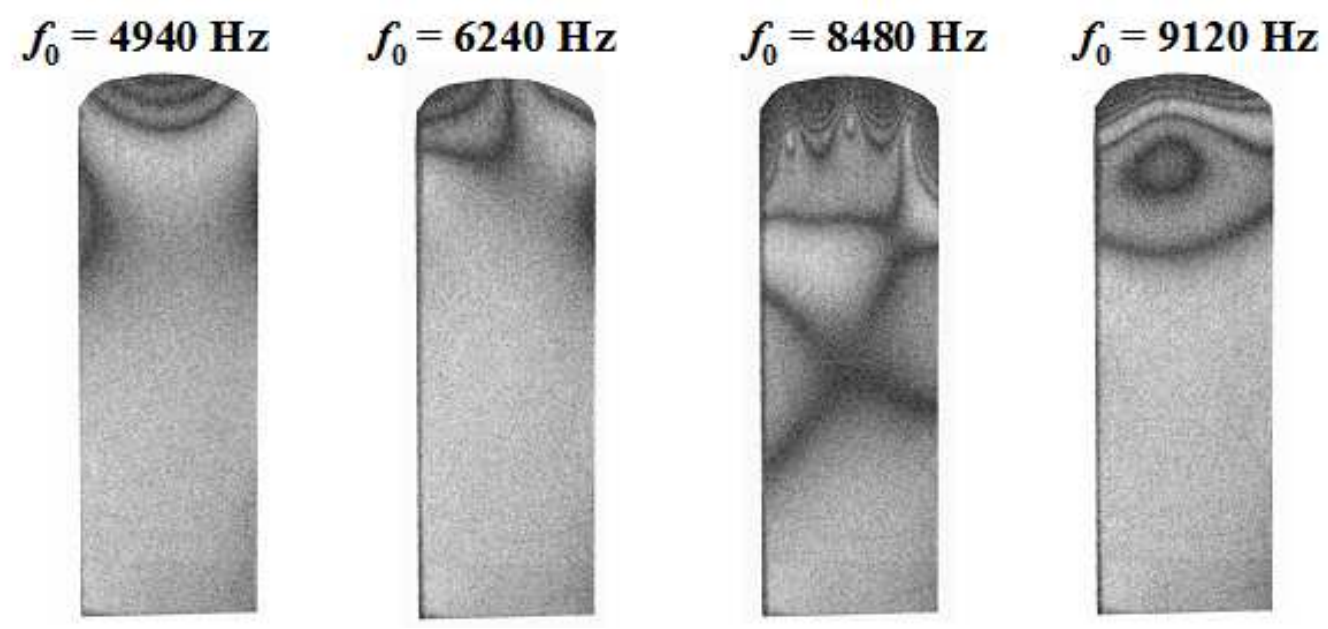

Figure 3 


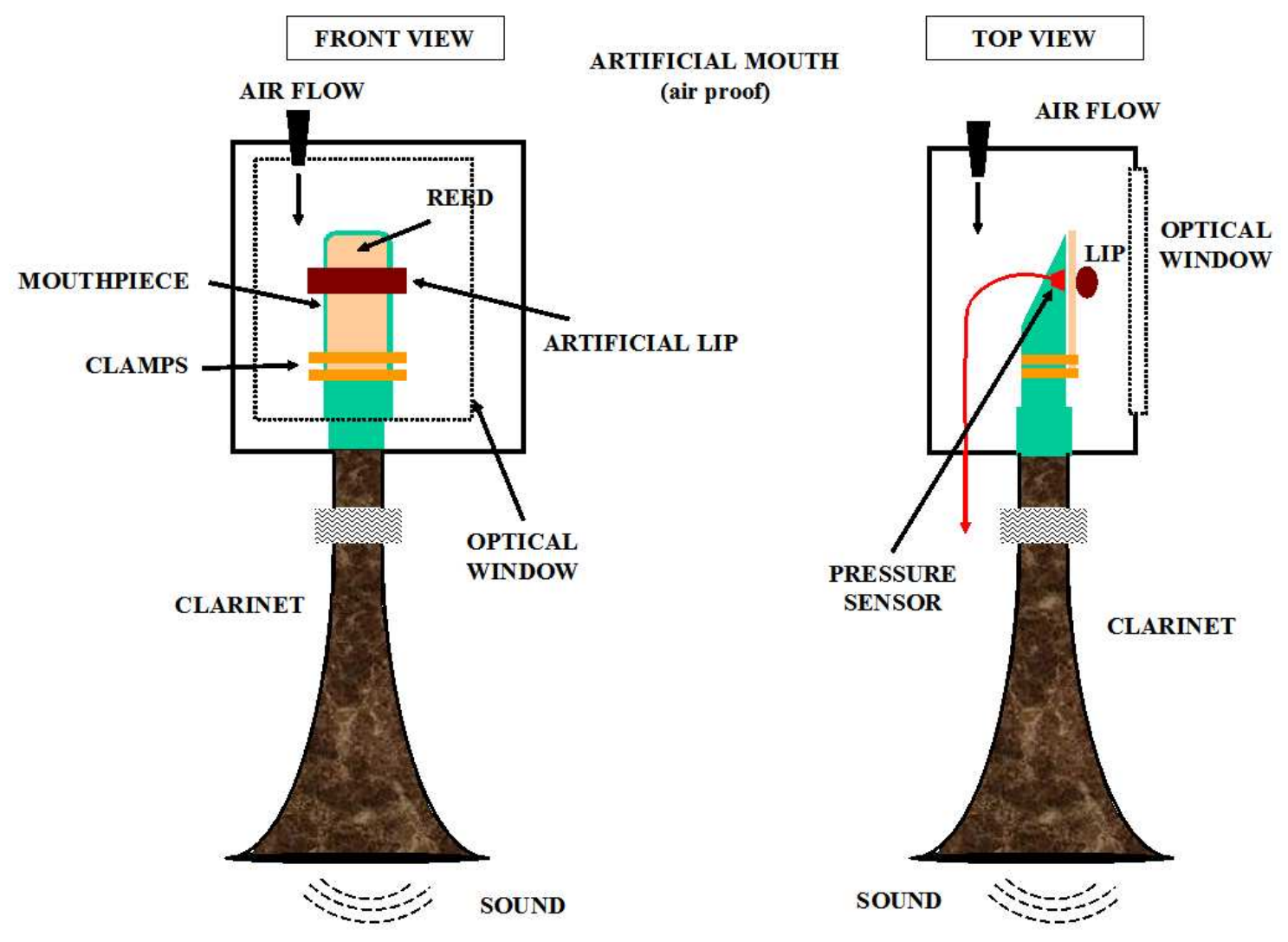

Figure 4 


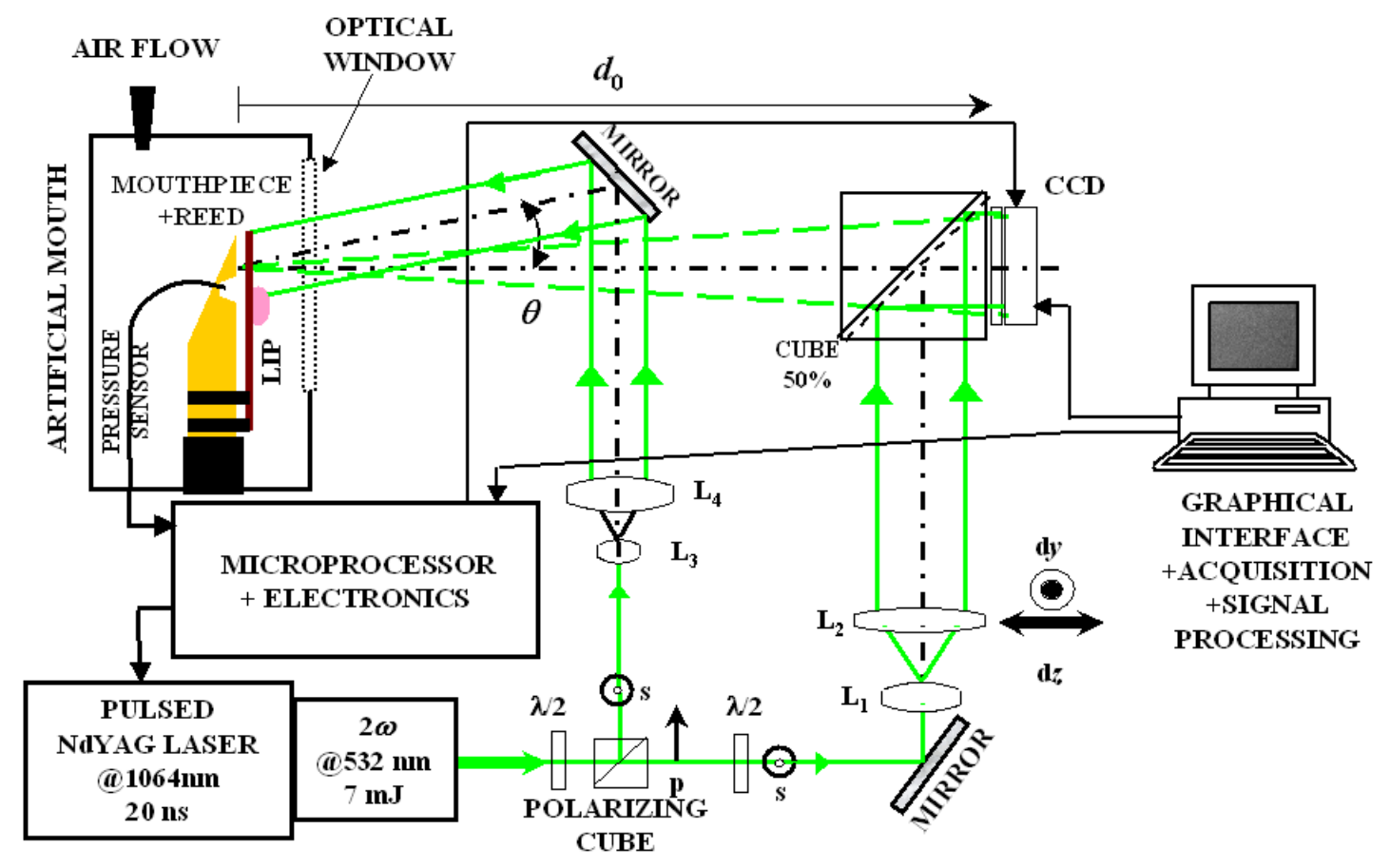

Figure 5 


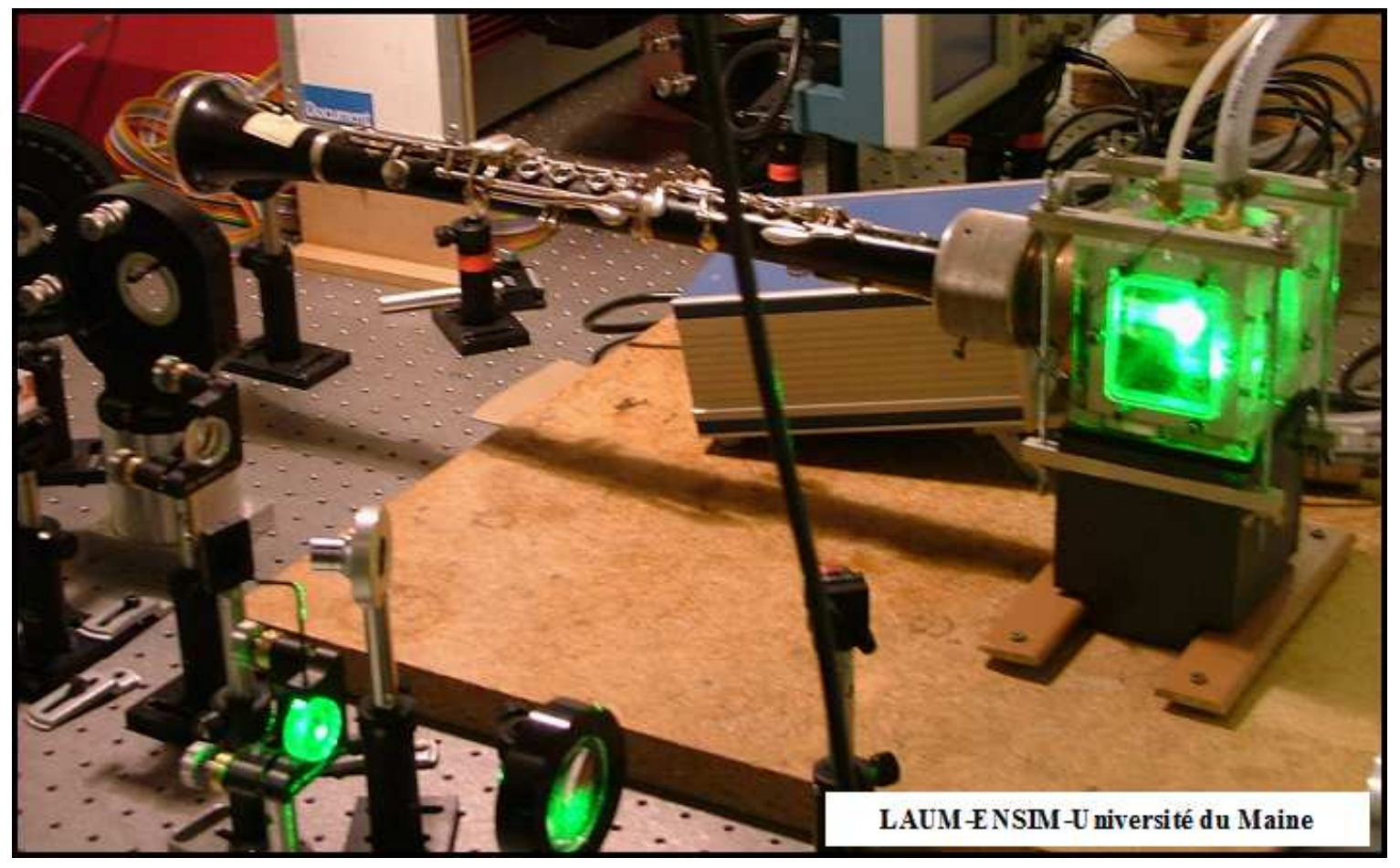

Figure 6 


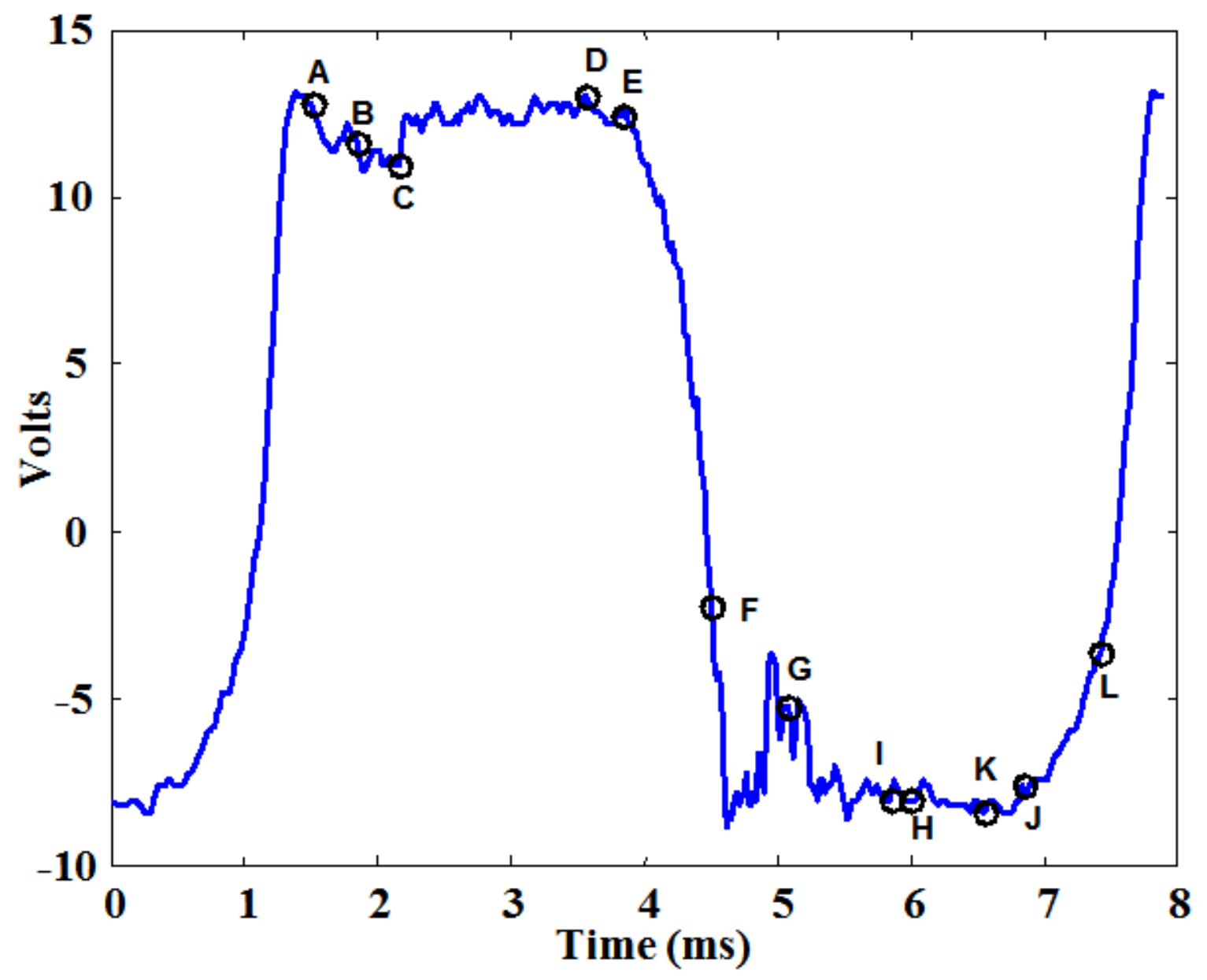

Figure 7 


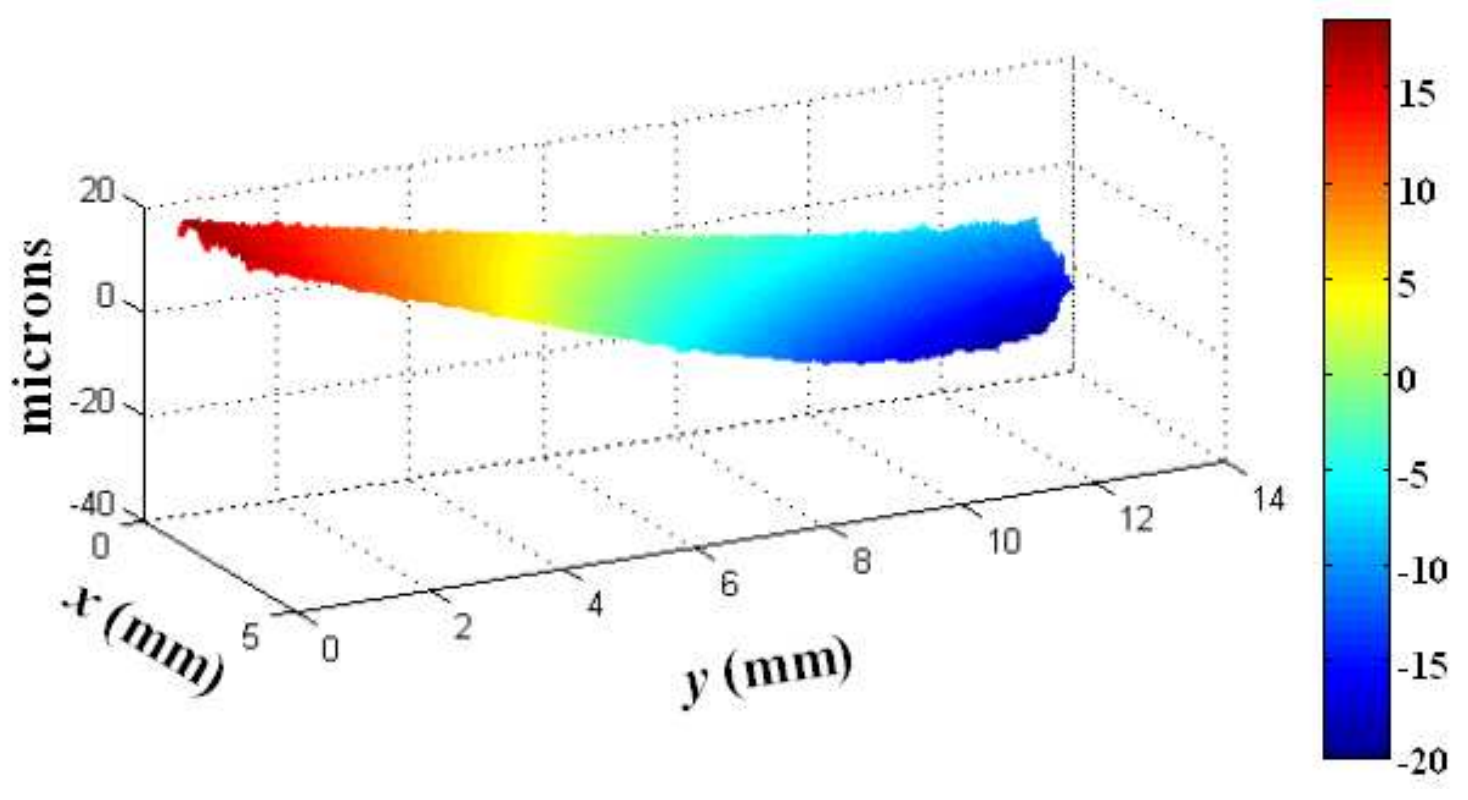

Figure 8 


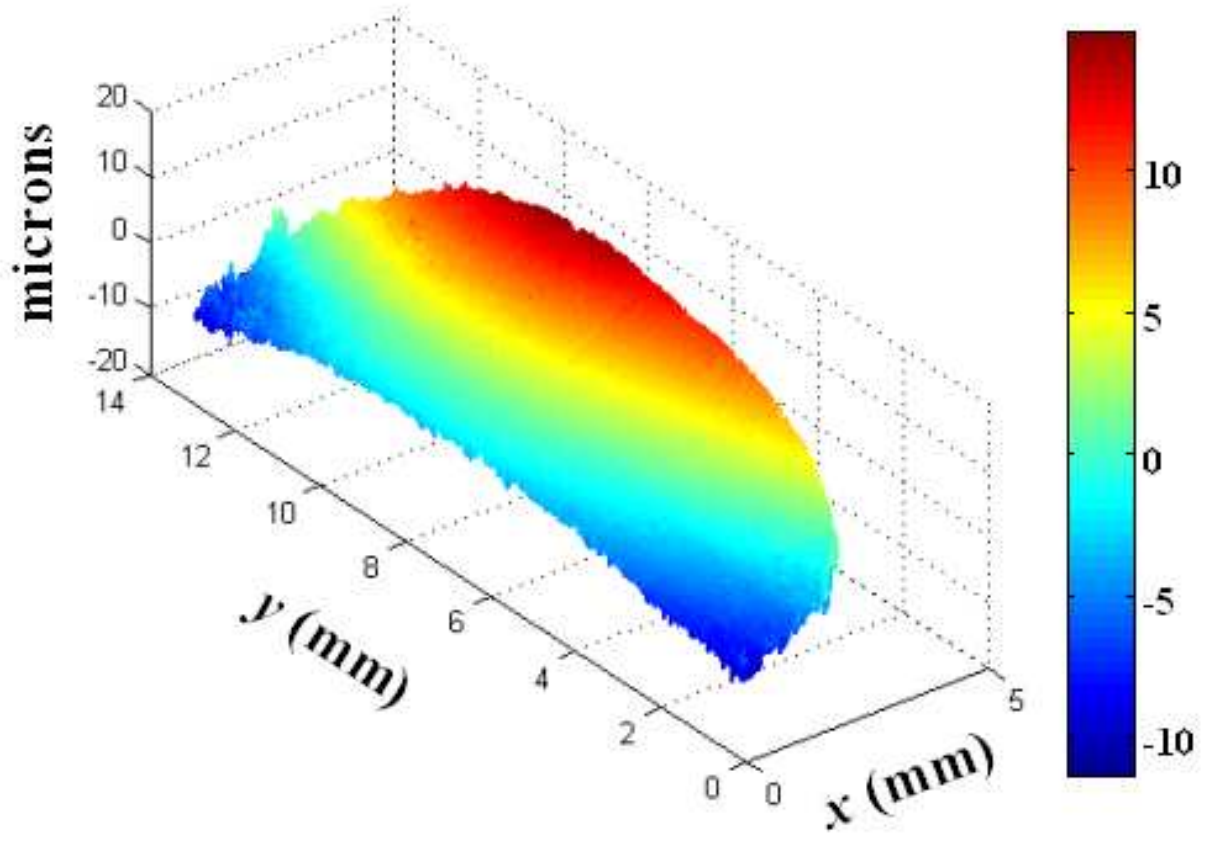

Figure 9 

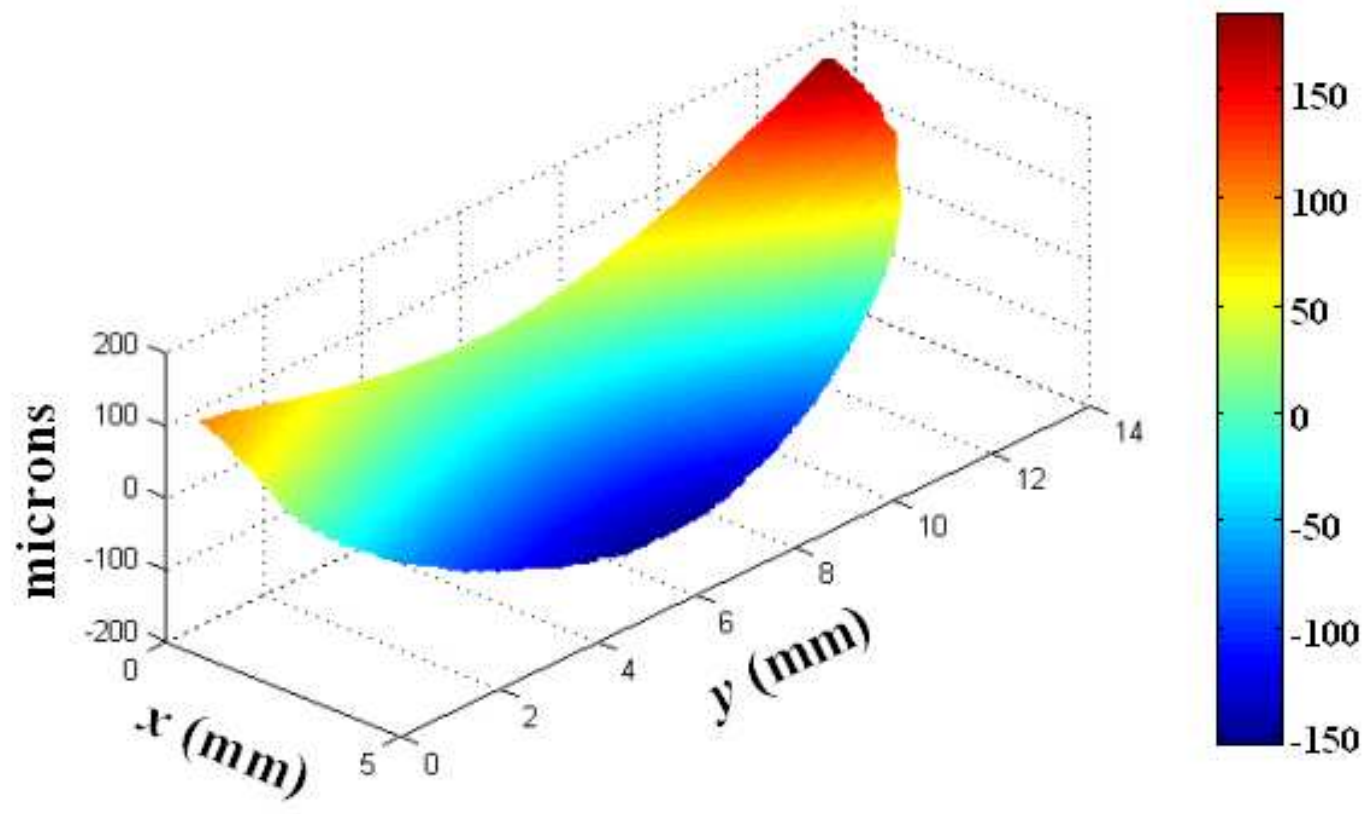

Figure 10 


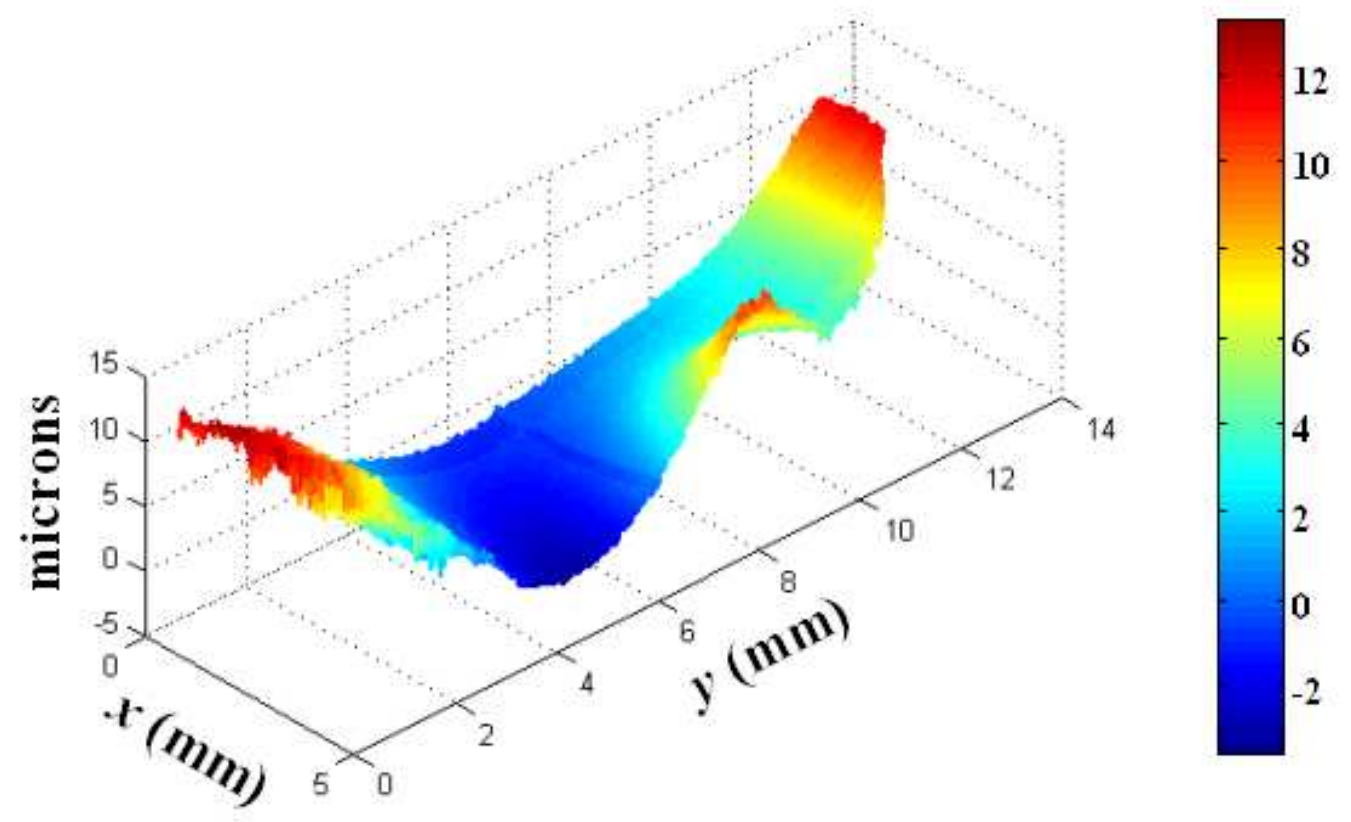

Figure 11 


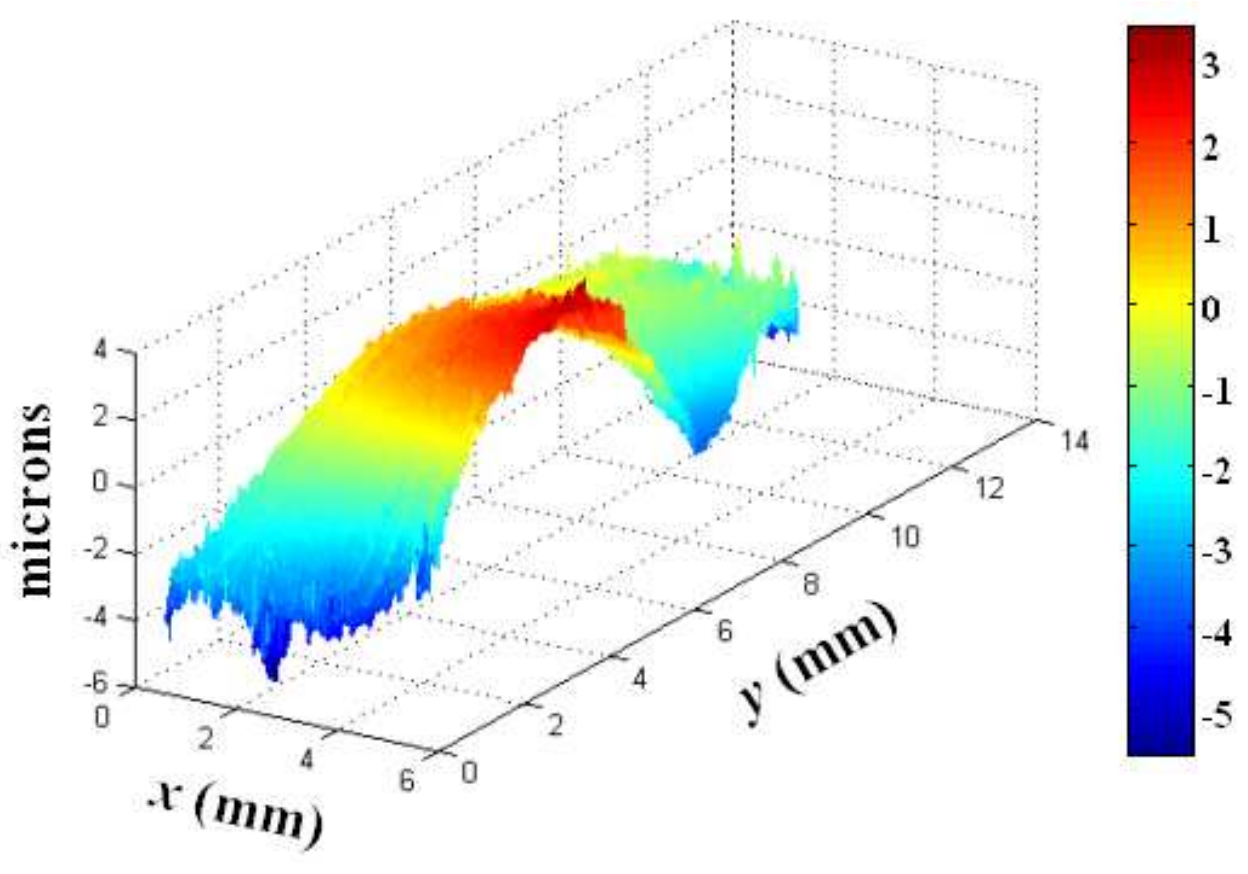

Figure 12 


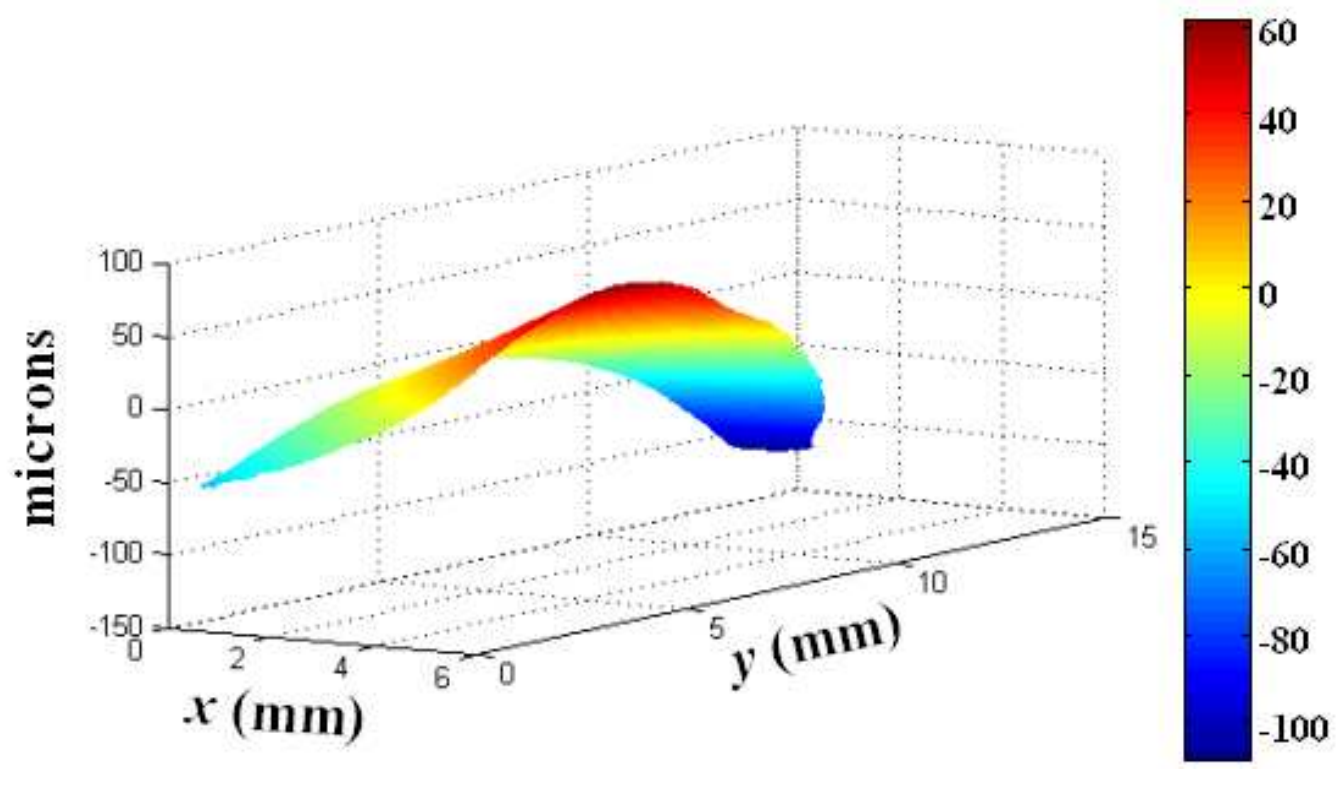

Figure 13 